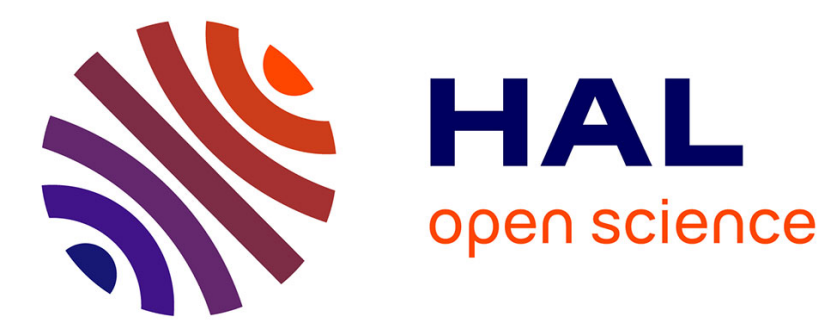

\title{
Sleepiness detection on read speech using simple features
}

Vincent P. Martin, Jean-Luc Rouas, Pierre Thivel, Jarek Krajewski

\section{To cite this version:}

Vincent P. Martin, Jean-Luc Rouas, Pierre Thivel, Jarek Krajewski. Sleepiness detection on read speech using simple features. 10th Conference on Speech Technology and Human-Computer Dialogue - SpeD 2019, Oct 2019, Timisoara, Romania. 10.1109/SPED.2019.8906577 . hal-02132438v4

\section{HAL Id: hal-02132438 \\ https://hal.science/hal-02132438v4}

Submitted on 10 Oct 2019

HAL is a multi-disciplinary open access archive for the deposit and dissemination of scientific research documents, whether they are published or not. The documents may come from teaching and research institutions in France or abroad, or from public or private research centers.
L'archive ouverte pluridisciplinaire HAL, est destinée au dépôt et à la diffusion de documents scientifiques de niveau recherche, publiés ou non, émanant des établissements d'enseignement et de recherche français ou étrangers, des laboratoires publics ou privés. 
archives-ouvertes

\section{Sleepiness detection on read speech using simple features}

Vincent P. Martin, Jean-Luc Rouas, Pierre Thivel, Jarek Krajewski

\section{To cite this version:}

Vincent P. Martin, Jean-Luc Rouas, Pierre Thivel, Jarek Krajewski. Sleepiness detection on read speech using simple features. 2019. hal-02132438v4

\section{HAL Id: hal-02132438 \\ https://hal.archives-ouvertes.fr/hal-02132438v4}

Submitted on 10 Oct 2019

HAL is a multi-disciplinary open access archive for the deposit and dissemination of scientific research documents, whether they are published or not. The documents may come from teaching and research institutions in France or abroad, or from public or private research centers.
L'archive ouverte pluridisciplinaire HAL, est destinée au dépôt et à la diffusion de documents scientifiques de niveau recherche, publiés ou non, émanant des établissements d'enseignement et de recherche français ou étrangers, des laboratoires publics ou privés. 


\section{Sleepiness detection on read speech using simple features}

\author{
Vincent P. Martin \\ LABRI- CNRS - UMR 5800 \\ Univ. Bordeaux - Bordeaux INP \\ Talence, France \\ vincent.martin@labri.fr \\ Jarek Krajewski \\ Engineering Psychology \\ Rhenish University of Applied Science \\ Cologne, Germany \\ j.krajewski@ixp-wuppertal.de
}

\author{
Jean-Luc Rouas \\ LABRI- CNRS - UMR 5800 \\ Univ. Bordeaux - Bordeaux INP \\ Talence, France \\ rouas@labri.fr
}

\author{
Pierre Thivel \\ LaBRI- CNRS - UMR 5800 \\ Univ. Bordeaux - Bordeaux INP \\ Talence, France \\ pierre.thivel@ensea.fr
}

\begin{abstract}
This paper is about automatic sleepiness state detection using speech samples. Following previous research carried out for the Interspeech 2011 challenge, we use the Sleepy Language Corpus (SLC) for our experiments. However, as we are willing to record our own subjects within a collaboration project with the Bordeaux hospital, we focus only on the read speech samples of that database. Furthermore, we are looking for understandable cues that can guide clinicians to provide a diagnostic. Hence, we devised a set of meaningful features that are close to the signal and restrict the feature selection process to methods that do not use feature combinations. Thus, using simple correlations and a grid search procedure on the training and development parts of the database, we selected a final $s$ et of 23 f eatures, $r$ eaching a performance on par with state-of-the-art systems. A discussion is proposed on the subjective ground truth used for the boundary between sleepy and non sleepy speech in this database. Finally, we discuss on the interpretation of the features and provide hints on the physiological causes.
\end{abstract}

Index Terms-Sleepiness detection, feature selection, prosody, read speech

\section{INTRODUCTION}

One of the major challenges for treating neuro-psychiatric pathologies is the follow-up of chronic patients in order to measure early relapses as well as observance and compliance to the treatment. Such a monitoring is possible thanks to connected medical devices (measuring for instance weight, blood pressure or physical activities) but crucial information about how the patients feels are difficult to measure. Regular in-person appointments between doctors and patients are thus required. The growing number of patients however increases

This work is carried out in the framework of the IS-OSA project funded by the French Region Nouvelle Aquitaine. This work was partially supported by the Horizon H2020 My Active and Healthy Ageing, (No. 689592) and German Federal Ministry of Education and Research (BMBF) under grant agreement 01-S15024 (VIVID - IKT2020/2015-2018). the queuing time and often results in episodic followups with unevenly spaced interviews.

Apart from the clinical interviews, it is nonetheless possible to measure some symptoms (e.g.: sadness or sleepiness) with a range of techniques: looking at eye movements, measuring electroencephalographic responses, or examining verbal expressions or body movements. Thanks to recent advances in speech processing, it is now possible to detect precise cues in the voice allowing to characterise the state of a speaker (e.g. to measure the sleepiness level). This method has the following advantages: recording voice data is not invasive and does requires neither specific sensors nor complex calibration processes. It can thus be set up in various open environments, outside laboratories, and allows regular and non-restrictive monitoring of patients.

Studies on sleepiness detection through voice has seen a peak of interest in the early 2010s, culminating with the 2011 Interspeech challenge [1]. Since then, there have been only few reported research on the subject [2]. The best performing automatic classification approach is still a system proposed in 2011 [3] and shares the same feature set as most research [4][6] computed with openSmile [7]. While some have tried to suggest using different features [8], [9], the common drawback to all of these systems is the lack of possible physiological interpretation of the features due to feature combination techniques.

We intend to provide a decision on the sleepiness level of the speaker, but that decision should be justified by the observation of specific traits in the voice that may have to be confirmed by clinicians. Thus, instead of designing new classifiers or new features selection processes, we wish to investigate in this paper if good classification performances can be obtained using a set of simple features that could be interpretable for clinicians. 
This paper is structured as follows. In Section 2, we provide a brief description of the Sleepy Language Corpus. In Section 3 , we introduce a description of our Custom Set of Features (CSF) and Section 4 presents the systems using them. Section 5 provides physiological interpretation of the results. Finally, Section 6 gives a conclusion and perspective on the subject.

\section{DATABASE PRESENTATION}

\section{A. Sleepy Language Corpus}

The SLC (Sleepy Language Corpus) is the reference corpus for the Interspeech 2011 challenge [1] on detection of sleepiness through voice. This database is composed of multiple speech tasks conducted in parallel of other sleeping-deprivation studies. The speakers are German volunteers and all the speech samples are in German language. More information about the experimental setup of the recordings can be found in [10], [11]. For extensive details about the dataset and the different experiments composing it, we invite the reader to see [12].

The additional information given in the database are the task, genre, attribution in train-development-test set and the mean of three Karolinska Sleeping Scale (KSS) scores [13, p.209]. KSS is a subjective sleepiness scale ranging from 1 (very alert) to 9 (sleepy with difficulties to stay awake): the score used in the database is the mean of the perceptual value filled by the speaker and the score of two external observers trained to evaluate behavorial sleepiness.

\section{B. Selection of a read subset of the database}

This research is a preliminary study for a project aiming at providing followup for patients treated for sleepiness problems at the Sleep Clinic of Bordeaux ${ }^{1}$. Since our target population may already be under treatment and have interaction difficulties, we decided to consider only a reading task which have a light cognitive load [14]. Furthermore, we selected only the reading tasks with an average duration over 8 seconds: the reading of the fable "Nordwind und Sonne" ("The Northwind and the Sun") (mean duration: 36.5 seconds for northw), the reading of two simulated air traffic control communications (mean duration: $9.7 \mathrm{~s}$ for flight1 and $13.8 \mathrm{~s}$ for flight2) and the reading of a simulated air traffic controller sentence (mean duration: $8.5 \mathrm{~s}$ for rogerl).

\section{Ground truth: The Karolinska Sleeping Scale}

The KSS being a semi-continuous measure, the choice is made to split the dataset into two classes: following [4], [6], [9], [15], the samples with a mean $\mathrm{KSS}>7.5$ will be considered as Sleepy Language (SL). On the contrary, the samples with a $\mathrm{KSS} \leq 7.5$ are labelled as Non Sleepy Language (NSL). Some statistics of the database with the KSS limit fixed at 7.5 are presented in Table I. It should be mentioned that others choices

\footnotetext{
${ }^{1}$ Centre Hospitalier Universitaire de Bordeaux
}

for that boundary may be made, such as a limit of 7 as in [16] or 8 in [10].

However, setting the limit between sleepy and non-sleepy is an arbitrary choice that may be different according to sleepiness specialists. We will further comment on this limit in the discussions on the results in Section IV-D.

TABLE I

STATISTICS: NUMBER OF SAMPLES AND TOTAL DURATION OF THE SELECTED READ PARTS OF THE SLC DATABASE WITH KSS LIMIT FIXED AT 7.5.

\begin{tabular}{|l|l|l|l|l|l|}
\hline Samples & & Train & Dev & Test & All \\
\hline Male & \multirow{2}{*}{ NSL } & 58 & 36 & 60 & 154 \\
& & $1089 \mathrm{~s}$ & $680.5 \mathrm{~s}$ & $1035.3 \mathrm{~s}$ & $1872.8 \mathrm{~s}$ \\
\cline { 2 - 6 } & SL & 29 & 47 & 22 & 98 \\
& & $364.9 \mathrm{~s}$ & $680.5 \mathrm{~s}$ & $297.0 \mathrm{~s}$ & $2804.8 \mathrm{~s}$ \\
\hline Female & NSL & 119 & 101 & 93 & 313 \\
& & $1624.6 \mathrm{~s}$ & $1286.6 \mathrm{~s}$ & $1340.7 \mathrm{~s}$ & $1342.4 \mathrm{~s}$ \\
\cline { 2 - 6 } & SL & 96 & 63 & 66 & 225 \\
& & $941.7 \mathrm{~s}$ & $753.7 \mathrm{~s}$ & $806.9 \mathrm{~s}$ & $4251.9 \mathrm{~s}$ \\
\hline Total & & 303 & 247 & 241 & 791 \\
& & $4019.6 \mathrm{~s}$ & $2720.8 \mathrm{~s}$ & $3478.9 \mathrm{~s}$ & $10219.3 \mathrm{~s}$ \\
\hline
\end{tabular}

\section{FEATURES EXTRACTION}

The goal of the project is to focus on features that can be understood and used by physicians. As a consequence, several features are extracted on two time scales. On one hand, excerpt level features are computed directly on each recording, using either an automatic vocalic segments detection algorithm [17] or voiced segments detected using a fundamental frequency extraction algorithm [18]. On the other hand, other features are computed on each voiced segment to characterise the regularity of production of harmonic sounds. These features are averaged for each recording.

\section{A. Excerpt level features}

The statistics on the duration/proportion of voiced segments or automatically detected vowels should reflect the global behaviour of the speaker. An example of excerpt level feature extraction is given on Figure 1.

The features extracted using this time-frame paradigm are:

- durvoiced: the total duration of voiced parts (in s.);

- pervoiced: the percentage in duration of voiced parts;

- durvowel: the total duration of vocalic segments (in s.);

- pervowel: the percentage in duration of vocalic segments.

This feature set provides 4 features per recording.

\section{B. Voiced segments features}

The voiced segment feature extraction is illustrated on Figure 2. These features include measurements on the fundamental frequency and intensity curves:

- FOMEAN: mean of fundamental frequency over a voiced segment; 


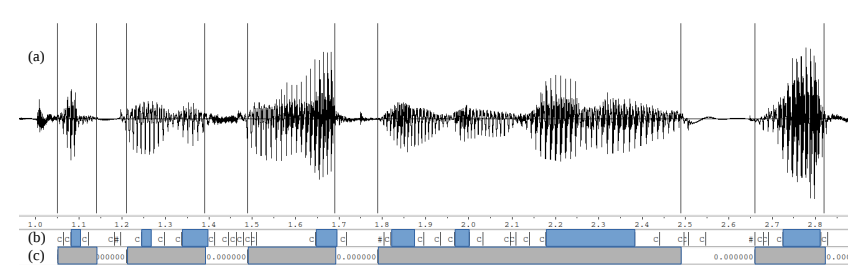

Fig. 1. Illustration of the result of the pre-processing steps for excerpt level features on the sentence "... sich Nordwind und Sonne, wer...": (a) signal, (b) vocalic segments (c) voiced segments.

- FOVAR: variance of fundamental frequency over a voiced segment;

- FOSLOPE: slope of the linear approximation of the fundamental frequency over a voiced segment;

- FOMAX: maximum of fundamental frequency over a voiced segment;

- FOMIN: minimum of fundamental frequency over a voiced segment;

- FOEXTEND: extend of fundamental frequency values over a voiced segment.

The same features are computed on the intensity curve (NRJMEAN, NRJVAR, NRJMAX, NRJMIN, NRJEXTEND). This results in 12 more features (6 on F0, 6 on intensity). We also computed the FOMEAN, FOVAR, NRJMEAN and NRJVAR features on vocalic segments, adding 4 features to the set.
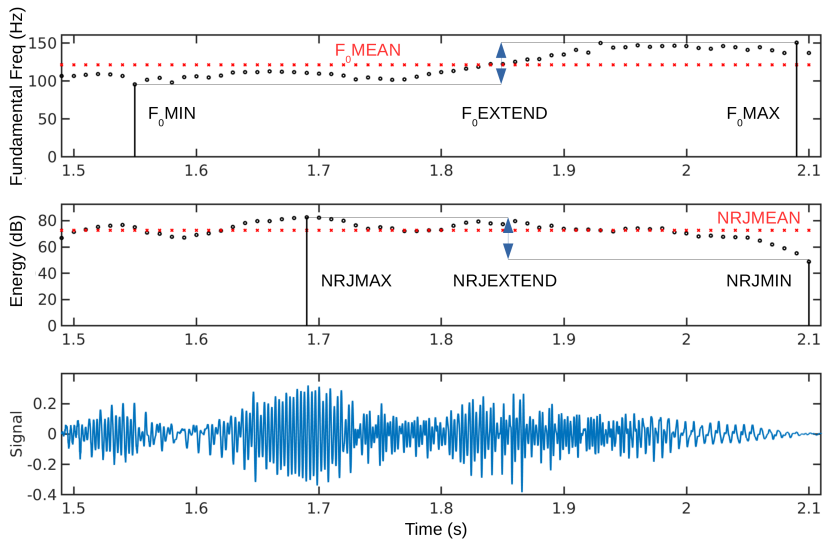

Fig. 2. Illustration of the extraction of features on a voiced segment. Upper pane: fundamental frequency in $\mathrm{Hz}$. Middle pane: intensity in $\mathrm{dB}$. Bottom pane: signal.

Furthermore, additional features are computed using the COVAREP matlab toolkit [19] which we modified to add some features and compute them only on voiced segments. These features have already been used for characterising singing styles [20] and for spoken social attitudes classification [21].They are the following ones: harmonics amplitude ( $\mathrm{H} 1$, $\mathrm{H} 2, \mathrm{H} 4)$, formants amplitude (A1, A2, A3), frequencies (F1, F2, F3, F4) and bandwidth (B1, B2, B3, B4), differences between harmonics amplitude (H1-H2, H2-H4), differences between amplitude of harmonics and formants (H1-A1, H1-
A2, H1-A3), cepstral peak prominence (CPP), harmonics to noise ratios on different frequency bands (HNR05, HNR15, HNR25, HNR35). All these features are averaged over each recording, yielding an additional set of 24 features per recording. We thus extract a total of 44 features.

\section{IS11 openSMILE features}

For comparison purposes, we also extracted the most widely adopted feature set consisting of 59 low-level descriptors (4 energy related descriptors, 50 spectral descriptors and 5 voice related descriptors), combined with 33 base functionals and 5 F0 functionnals, leading into 4368 features. A more complete description of these features is presented in [1].

\section{PRoposed Systems}

\section{A. Feature selection by statistical methods and SVM}

Before further analysis, all the features are centered with the mean values of the features for all the samples of a given speaker (number of speech samples for each speaker: mean $=13.4$, std=19.4).

In our framework, the reduction of the number of features is constrained by two limits. First, we do not wish to carry out dimensional reduction methods that lead to incomprehensible features such as Principal Components Analysis or Linear Discriminant Analysis, as our goal is to link sleepiness to vocal physiological events. Second, not only the reliability of the KSS as a measure of sleepiness is not certain but it is a semi-continuous measure: a threshold has to be set to label the samples into the sleepy class (SL) or non-sleepy class (NSL). This uncertainty encourage us to prefer methods that do not use a strict limit for the KSS.

One way to choose the features is to select those who have the highest correlation to the KSS measures and give good classification results. After a Shapiro test that ensure that the data is not normally distributed, we conduct a Spearman $\rho$ test to measure the correlation between each of the features and the KSS values. This computation is done only on the aggregated train + dev.

Then, we perform a grid search on the two parameters of the system: the KSS limit and the number of features. For each number $n$ of features, we select the $n$ features that correlate the most with the KSS. The labels are then determined according to the various possible KSS limits (between 5: "neither sleepy neither awaken") and 9: "very sleepy with great efforts to stay awake"). A Support Vector Machine (SVM) using the Radial Basis Function (RBF) kernel is implemented with the Python library sklearn [22] and trained using the training set and tested against the development set. Finally, for a given set of $n$ features, the reported result will be the best result over all the KSS limit values. The flowchart of the features selection is presented in Figure 3. This procedure is carried out using 
SVM RBF kernel $(\mathrm{C}=1)$

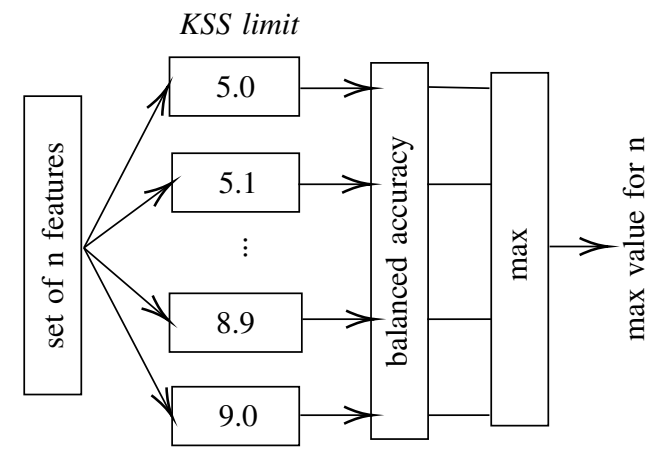

Fig. 3. Flowchart of the gridsearch to compute the best performance with $n$ features. The SVM are trained with the train dataset and tested with the development dataset.

our complete set of 44 features and the 200 IS11 openSMILE features that correlate the most with the KSS score.

The result of this experiment is shown on Figure 4. On the development set, the best performances are obtained using 23 features from our set (a) and 101 for the openSMILE features (b). They resp. reach an Unweighted Average Recall (UAR) of $68.1 \%$ and $70.3 \%$. Even though we do not report the results here, the performances obtained using the openSMILE features do not evolve significantly from 120 features on (we tested using up to 200 features).

The selected 23 features from our set are presented in Table II with their Spearman correlation value, their significance and their order of importance.

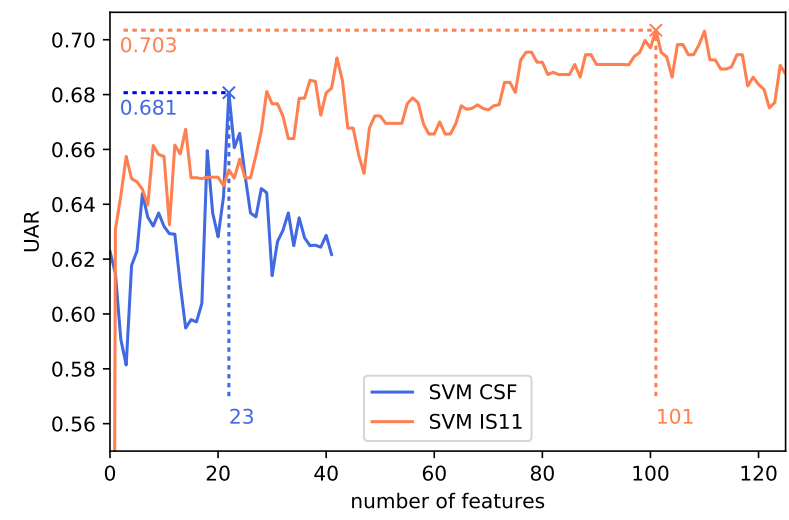

Fig. 4. Unweighted Average Recall (UAR) for the $n$ features that correlate the most with the KSS. The KSS limit is varying from 5 to 9: for each set of features, only the performance of the best system is kept.

\section{B. ASIMPLS}

Based on the Partial Least Square (PLS) algorithm [23], the ASIMPLS classifier achieve the best results of the state of the art when fused with SVM [4]. The latter article discusses, assuming few hypothesis, that the ASIMPLS has the advantage
TABLE II

THE SELECTED 23 FEATURES ACHIEVING THE BEST PERFORMANCES, THEIR SPEARMAN $\rho$, P-VALUE AND RANK.

$$
p<0.05:^{*}, p<0.01:^{* *}, p<0.001: * * *
$$

\begin{tabular}{|c|c|c|c|}
\hline Features & Spearman $\rho$ & $\mathrm{p}$ & $\mathrm{rank}$ \\
\hline durvoiced & 0.06 & 0.17 & 23 \\
durvowel & 0.05 & 0.29 & 19 \\
\hline F0 Mean (vowels) & -0.32 & $* * *$ & 1 \\
F0 Mean & -0.27 & $* * *$ & 2 \\
F0 Slope & -0.09 & $*$ & 16 \\
F0 Min & -0.20 & $* * *$ & 4 \\
F0 Max & -0.24 & $* * *$ & 3 \\
F0 Extend & -0.08 & 0.06 & 17 \\
\hline Energy Var (vowels) & -0.07 & 0.1 & 21 \\
Energy Var & -0.07 & 0.1 & 22 \\
Energy Slope & 0.13 & $* *$ & 10 \\
Energy Min & 0.14 & $* * *$ & 8 \\
Energy Extend & -0.16 & $* * *$ & 6 \\
\hline H1 & 0.10 & $*$ & 14 \\
H2 & 0.13 & $* *$ & 9 \\
A2 & -0.08 & 0.07 & 18 \\
A3 & -0.10 & $*$ & 13 \\
F1 & -0.19 & $* * *$ & 5 \\
B1 & -0.10 & $*$ & 15 \\
\hline H1A1 & 0.07 & $*$ & 20 \\
H1A2 & 0.12 & $* *$ & 11 \\
H1A3 & 0.14 & $* * *$ & 7 \\
\hline HNR05 & -0.12 & $* *$ & 12 \\
\hline
\end{tabular}

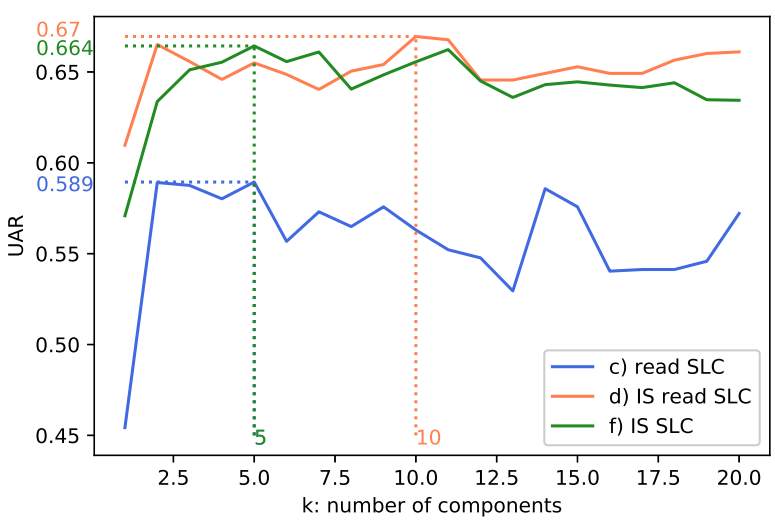

Fig. 5. Performances of the ASIMPLS systems with the best $b^{\prime}$ depending on the number of components $k$ on train vs dev

of taking the different speaking styles into account: speaker normalisation is not needed in this framework.

The two parameters of the ASIMPLS classifier are the number of components $k$ and the correction term $b^{\prime}$. The tuning procedure is the following. First, the features are scaled and normalised. Second, $k$ and $b^{\prime}$ are tuned with the train vs dev paradigm and we choose the parameters achieving the best accuracy with a KSS limit of 7.5. The result of the selection of $k$ is presented in Figure 5. 
The two best systems on train vs dev are obtained for IS11 openSMILE features, on the whole SLC $\left(\mathrm{k}=5, b^{\prime}=92.8 \times\right.$ $10-3, \mathrm{UAR}=66.4 \%)$ and the reading tasks $\left(\mathrm{k}=10, b^{\prime}=\right.$ $64.7 \times 10^{-3}$, UAR $\left.=67.0 \%\right)$, respectively noted $(\mathrm{f})$ and $(\mathrm{d})$. We also consider the system using our Custom Set of Features that achieves an UAR of $58.9 \%$ with $\mathrm{k}=5$ components and $b^{\prime}=4.5 \times 10^{-3}$, noted c). The results of trained on train + dev and tested on test are reported in Table III.

\section{Results using the conventional KSS limit}

The results of the different systems on train+dev vs test for a KSS limit of 7.5 are aggregated in the Table III. On the reading tasks, the best system is the SVM with the custom set of features (a), with an UAR of $76.39 \%$. We also report the performances achieved by the same system (23 features of our CSF + SVM) trained and tested on the whole SLC (e) for comparison purpose. The latter are worst than the ones achieved on the read tasks. These being the longest of the SLC, we assume that the other samples are too short to embrace the complexity of the sleepiness state detection problem. To discuss this hypothesis, we plot in Figure 6 the performances of the system (e) trained and tested on a subset of the whole SLC, depending on the minimal length of the samples. The trend shows that the longer the samples are, the better the UAR. The decrease of the performances observed from $9 \mathrm{~s}$ is due to the low number of samples classified as SL, leading to a decrease of the UAR. This result confirms our hypothesis that the lower performances of our system on the whole SLC compared with the read subset of the corpus are due to the too little length of the samples that does not come from read speech.

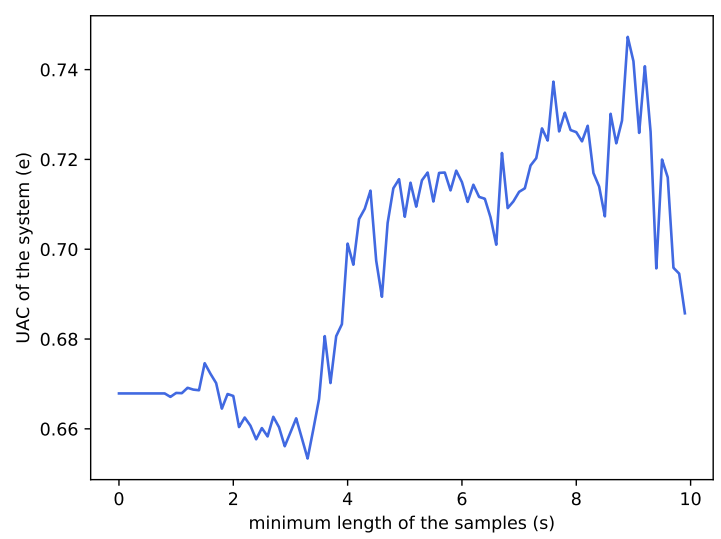

Fig. 6. UAR of the system (a) depending on the minimal length of the samples on the whole SLC.

On the contrary, the ASIMPLS algorithm achieves better performances trained and tested on the whole SLC (f) than on read tasks. We hypothesise that the multiplicity of samples allows the algorithm to model with better precision the speakers vocal characteristics, leading to a better classification of the sleepiness state.

TABLE III

RESULTS OF THE SYSTEMS ON train+dev VS test FOR KSSLIMIT=7.5.

\begin{tabular}{|c|c|c|c|c|}
\hline Ref & Features (\#) & Sensitivity & Specificity & UAR \\
\hline \multicolumn{5}{|c|}{ Reading SLC (241 samples) } \\
\hline a) & SVM CSF (23) & $75 \%$ & $77.78 \%$ & $76.39 \%$ \\
\hline b) & SVM IS11 (101) & $54.5 \%$ & $83.0 \%$ & $68.8 \%$ \\
\hline c) & ASIMPLS CSF (5) & $44.3 \%$ & $83.0 \%$ & $63.7 \%$ \\
\hline d) & ASIMPLS IS11 (10) & $56.8 \%$ & $78.4 \%$ & $67.6 \%$ \\
\hline \multicolumn{5}{|c|}{ Entire SLC database (2808 samples) } \\
\hline e) & SVM CSF (23) & $40.0 \%$ & $73.5 \%$ & $66.8 \%$ \\
\hline \multirow[t]{2}{*}{ f) } & ASIMPLS IS11 (5) & $67.8 \%$ & $68.8 \%$ & $68.3 \%$ \\
\hline & State of the art [4] & $64.3 \%$ & $79.1 \%$ & $71.7 \%$ \\
\hline
\end{tabular}

\section{Sensitivity of the system to the KSS limit}

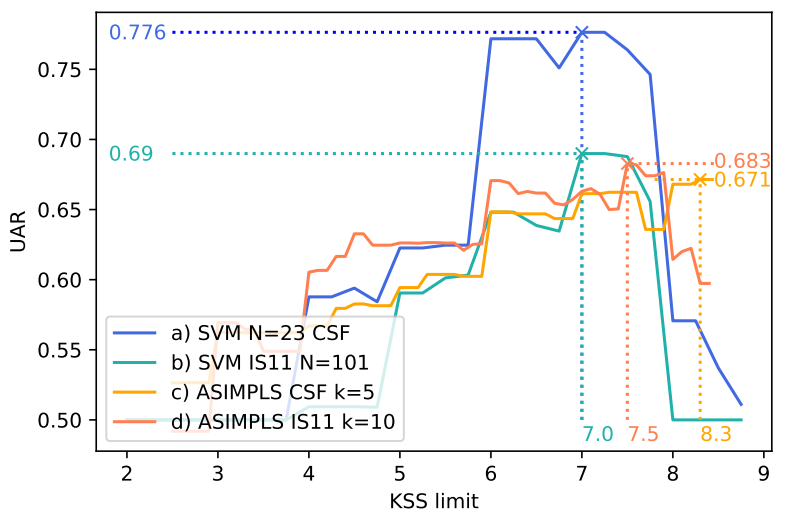

Fig. 7. Sensitivity of the system to the limit of the KSS on the read tasks of the SLC.

Although most researches use a KSS limit fixed at 7.5, a clear consensus on this value does not seems to exist. Furthermore, fixing a threshold between Sleepy and Non Sleepy patients may be a question left to the appreciation of a specialist.

To answer this problem, we compute for different KSS limits the UAR of the output of the system, keeping identical all other parameters, using the same method as in section IV-C. The results on the read tasks of the SLC are presented on Figure 7.

Even if the KSS limit of 7.5 leads to satisfying results, the best results are obtained for both systems with a KSS limit of 7 , achieving respectively $77.6 \%$ and $69.0 \%$ of UAR using our set of features and the openSMILE features. Not only the proposed features show satisfying performances for a conventional KSS limit (7 or 7.5), but they are significant of a more global state of sleepiness, whatever the KSS limit being between 6 and 8. For a KSS limit near 6 (between 'Neither Awaken nor Sleepy' and 'Sleepy without efforts to maintain wakefulness'), the system identify the beginning of the sleepiness state. On the contrary, for a KSS limit near 8 
(between 'Sleepy without efforts to maintain wakefulness' and 'Sleepy fighting to stay awake'), the system identifies a state of advanced sleepiness. This study allows to let the specialised physicians to determine what state of sleepiness they need to evaluate, the proposed system achieving in any case relevant performances.

\section{PhysiologicAl INTERPRETATION}

One of the biggest constraint of this work is to select features that can relate the physiological modifications of the voice of the patient to sleepiness.

First, similarly to [24], an augmentation of the voiced and vowels parts is observed. This observation can be clue to the augmentation of hesitations of the sleepy speakers. The diminution of the values of F0 Mean, F0 Min, F0 Max, F0 Extend, F0 Slope, frequency F1 (also observed in [10] and [25]), bandwidth of F1 and amplitude of second and third formants witness a shift of the frequencies contained in the voice towards lower values. This accords with [26], [10] and [27]. Moreover, the diminution of the values of F0 Extend and F0 Slope are clues of a reduction of the bandwidth used during the vocal process.

Contrary to the observations made in [10], the energy extend, the absolute value of the energy slope and the variance of the energy decrease with sleepiness. Combined with the rise of the lower frequencies, the higher energies staying constant, these observations express a diminution of nuances in the Sleepy Language. We hypothesise that the slight augmentation of the first harmonic frequency, that seems contrary to previous observations, is due to the modification of the exhaled air flux that modifies the distribution of harmonics but not formants [28]. This is consistent with the diminution of the HNR (HNR05 in our case) also observed in [29].

All these observations lead to the hypothesis that the sleepy speakers struggle to produce the same variety of nuances of frequencies, energy and quality of voiced parts.

\section{Vi. Conclusion \& Perspectives}

In this paper, we have proposed a novel strategy for sleepiness detection in voice, with possible applications in the medical field. We have shown that the subset of reading tasks leads to better results for sleepiness detection and we have developed a set of adapted features. Our system performances are comparable to state of the art methods. The careful selection of features as well as the choice of the subset of the SLC enhance the detection of sleepiness through voice. Moreover, we have proposed a physiological analysis of the vocal parameters for various levels of sleepiness. In the future, we would like to apply these results on the elaboration of a new database in collaboration with the Bordeaux hospital.

\section{REFERENCES}

[1] B. Schuller, S. Steidl, A. Batlinder, F. Schiel, and J. Krajewski, "The INTERSPEECH 2011 Speaker State Challenge," in Interspeech, 2011.

[2] N. Cummins, A. Baird, and B. Schuller, "Speech analysis for health: Current state-of-the-art and the increasing impact of deep learning," Health Informatics and Translational Data Analytics, vol. 151, pp. 1-54, 2018.

[3] D.-Y. Huang, Y. Tsao, H. Chiori, and H. Kashioka, "Feature Normalization and Selection for Robust Speaker State Recognition," in IEEE International Conference on Speech Database and Assessments, 2011.

[4] D.-Y. Huang, Z. Zhang, and S. S. Ge, "Speaker State Classification Based on Fusion of Asymmetric Simple Partial Least Squares (SIMPLS) and Support Vector Machines," Comput. Speech Lang., vol. 28, no. 2, pp. 392-419, 2014.

[5] J. Krajewski, S. Schnieder, C. Monschau, R. Titt, and D. Sommer, "Large Sleepy Reading Corpus (LSRC): Applying Read and Speech for Detecting Sleepiness," Speech Communication; 12. ITG Symposium, pp. 1-4, 2016.

[6] Y. Zhang, F. Weninger, and B. Schuller, "Cross-Domain Classification of Drowsiness in Speech: The Case of Alcohol Intoxication and Sleep Deprivation," in Interspeech, 2017.

[7] F. Eyben and B. Schuller, "Opensmile," ACM SIGMultimedia Records, vol. 6 , pp. 4-13, 2015.

[8] J. Krajewski, S. Schnieder, D. Sommer, A. Batlinder, and B. Schuller, "Applying multiple classifiers and non-linear dynamics features for detecting sleepiness from speech," Neurocomputing, vol. 84, pp. 65-75, 2011.

[9] C. Sezgin, B. Günsel, and J. Krajewski, "Medium term speaker state detection by perceptually masked spectral features," Speech Communication, vol. 67, pp. 26-41, 2015.

[10] J. Krajewski, A. Batlinder, and M. Golz, "Acoustic sleepiness detection: Framework and validation of a speech-adapted pattern recognition approach," Behavior Research Methods, vol. 41, no. 3, pp. 795-804, 2009.

[11] M. Golz, D. Sommer, M. Chen, D. Mandic, and U. Trutschel, "Feature Fusion for the Detection of Microsleep Events," Journal of VLSI Signal Processing, vol. 49, pp. 329-342, 2007.

[12] B. Schuller, S. Steidl, A. Batlinder, F. Schiel, J. Krajewski, F. Weninger, and F. Eyben, "Medium-term speaker states-A review on intoxication, sleepiness and the first challenge," Comput. Speech Lang., vol. 28, no. 2, pp. 346-374, 2013.

[13] A. Shahid, K. Wilkinson, S. Marcu, and C. M. Shapiro, eds., STOP, THAT and One Hundred Other Sleep Scales. Springer-Verlag New York, 2011.

[14] G. Christodoulides, Effects of Cognitive Load on Speech Production and Perception. PhD thesis, Université Catholique de Louvain, 2016.

[15] B. Günsel, C. Sezgin, and J. Krajewski, "Sleepiness detection from speech by perceptual features," in IEEE - ICASSP, pp. 788-792, 2013.

[16] H. Martensson and O. Keelan, Feature Engineering and Machine Learning for Driver Sleepiness Detection. PhD thesis, 2017.

[17] F. Pellegrino and R. Andre-Obrecht, "Automatic language identification: an alternative approach to phonetic modelling," Signal Processing, vol. 80, no. 7, pp. 1231-1244, 2000.

[18] K. Sjölander, “The Snack Sound Toolkit,” 2004

[19] G. Degottex, J. Kane, T. Drugman, T. Raitio, and S. Scherer, "COVAREP - A collaborative voice analysis repository for speech technologies," in IEEE - ICASSP, pp. 960-964, 2014.

[20] J.-L. Rouas and L. Ioannidis, "Automatic Classification of Phonation Modes in Singing Voice: Towards Singing Style Characterisation and 
Application to Ethnomusicological Recordings," in Interspeech, pp. 150154, 2016.

[21] J.-L. Rouas, T. Shochi, M. Guerry, and A. Rilliard, "Categorisation of spoken social affects in Japanese: human vs. machine," in ICPhS, 2019.

[22] F. Pedregosa, G. Varoquaux, A. Gramfort, V. Michel, B. Thirion, O. Grisel, M. Blondel, P. Prettenhofer, R. Weiss, V. Dubourg, J. Vanderplas, A. Passos, D. Cournapeau, M. Brucher, M. Perrot, and E. Duchesnay, "Scikit-learn: Machine Learning in Python," Journal of Machine Learning Research, vol. 12, pp. 2825-2830, 2011.

[23] S. De Jong, "SIMPLS: An alternative approach to partial least squares regression," Chemometrics and Intelligent Laboratory Systems, vol. 18, no. 3, pp. 251-263, 1993.

[24] L. S. Dhupati, S. Kar, A. Rajaguru, and A. Routray, "A novel drowiness detection scheme based on speech analysis with validation using simultaneous EEG recordings," in IEEE - Int. CASE, pp. 917-921, 2010.

[25] H. P. Greeley, E. Friets, J. P. Wilson, S. Raghavan, J. Picone, and J. Berg, "Detecting Fatigue From Voice Using Speech Recognition," in IEEE International Symposium on Signal Processing and Information Technology, pp. 567-571, 2006.

[26] T. L. Nwe, H. Li, and D. Minghui, "Analysis and Detection of Speech under Sleep Deprivation," in Interspeech, 2006.

[27] E. L. McGlinchey, L. S. Talbot, K.-h. Chang, K. A. Kaplan, R. E. Dahl, and A. G. Harvey, "The Effect of Sleep Deprivation on Vocal Expression of Emotion in Adolescents and Adults," Sleep, vol. 34, pp. 1233-1241, 2011.

[28] J. Hillenbrand, R. Cleveland, and R. L. Erickson, "Acoustic correlates of breathy vocal quality," Journal of Speech, Language, and Hearing Research, vol. 37, no. 4, pp. 769-778, 1994.

[29] S. Boyer, R. El-Yagoubi, M. Tiberge, R. Ruiz, and A. Daurat, "Paramètres Acoustiques de la Voix et Privation de Sommeil," in CFA/VISHNO, 2016. 
